\title{
SOLUÇÕES AJUSTADAS DO FPO VIA MODELO PARAMETRIZADO
}

\author{
Carlos Fabian Moyano* \\ moyano@labspot.ufsc.br
}

\author{
Roberto de Souza Salgado* \\ salgado@labspot.ufsc.br
}

*Universidade Federal de Santa Catarina, CTC / EEL / LABSPOT, 88040-900, Florianópolis, Santa Catarina, Brazil

\begin{abstract}
This work presents a parameterized formulation of the Optimal Power Flow (OPF) problem, which aims at avoiding the divergence of the OPF iterative process. The objective function of the modified optimisation problem is given by the weighted sum of the original performance index to be optimized with the parameter applied to adjust the demand and/or limits. The proposed methodology provides indication of the potential causes of the non-convergence and the possible actions that could be taken to determine an operational solution. If the originally specified demand can be supplied satisfying the operational limits without load/limits adjustments, the proposed methodology determines the conventional OPF solution. Numerical results obtained with test systems of 6, 118 and 750 buses illustrate the application of the proposed strategy.
\end{abstract}

KEYWORDS: Optimal Power Flow, Parameterization, Interior Point Method.

\section{RESUMO}

Este trabalho apresenta uma formulação parametrizada do Fluxo de Potência Ótimo (FPO), a qual visa evitar a divergência do processo iterativo do FPO. A função objetivo do problema modificado é representada pela soma ponderada do índice a ser otimizado originalmente com o parâmetro de ajuste da carga e/ou limites. A metodologia proposta for-

\footnotetext{
Artigo submetido em 18/08/2005

1a. Revisão em 10/10/2005

2a. Revisão em 06/04/2006

Aceito sob recomendação do Editor Associado Prof. Carlos A. Castro
}

nece uma indicação das potenciais causas da divergência do processo iterativo e das possíveis medidas que podem ser tomadas para se obter uma solução operacional. No caso em que o carregamento originalmente especificado pode ser suprido sob condições operativas, a solução do problema de FPO convencional é determinada. Resultados numéricos obtidos com sistemas teste de 6,118 e 750 barras ilustram a aplicação da estratégia proposta.

PALAVRAS-CHAVE: Fluxo de Potência Ótimo, Parametrização, Método de Pontos Interiores

\section{INTRODUÇÃO}

A utilização plena dos aplicativos computacionais de Fluxo de Potência Ótimo não-linear na análise da operação dos sistemas de potência tem sido dificultada pela falta de confiabilidade na convergência do processo iterativo. Em particular, quando este processo diverge, freqüentemente não há disponibilidade de soluções alternativas para as equações da rede elétrica ou mesmo indicadores da causa da divergência.

A não-convergência do FPO é uma conseqüência da inexistência de uma solução que satisfaça simultaneamente as restrições de igualdade e desigualdade ou da impossibilidade de se alcançar o valor ótimo do índice de desempenho. Em ambos os casos, algumas matrizes relacionadas às condições de otimalidade tornam-se singulares no decorrer do processo iterativo. Quando o suprimento da demanda sob condições operacionais é inviável, a matriz Jacobiana das restrições de igualdade e desigualdade ativas tem posto deficiente e pelo menos um multiplicador de Lagrange tende a infinito. A não factibilidade da solução pode ser resultante de um nível de carregamento situado além do ponto bifurcação de sela-nó 
das equações que representam a rede elétrica (Dobson, 1992) ou de condições por demais restritivas na região definida pelos limites operativos (Greene et al., 2002).

A dificuldade de se atingir o ponto ótimo também pode ser oriunda da forma analítica da função objetivo. A determinação de pontos críticos de funções não lineares com mínimos locais muito próximos é consideravelmente complexa por causa das oscilações em torno das soluções ótimas. Quando esta situação ocorre, a projeção da matriz Hessiana no conjunto de restrições de igualdade e desigualdade ativas possui em geral um autovalor nulo.

O estudo da não convergência do processo iterativo do FPO pode ser realizado sob a ótica do máximo carregamento que o sistema pode suportar levando em consideração os limites operativos. Uma demanda para a qual não existe solução viável que satisfaça as restrições de desigualdade, pode ser interpretada como superior ao carregamento máximo operativo do sistema. A determinação deste carregamento é formulada em (Irisarri et al., 1997) como um problema de otimização estático não-linear, onde a demanda parametrizada de cada barra é maximizada numa direção de variação préespecificada. Outra forma de se tratar o problema de não convergência do FPO é através da restauração da solubilidade das equações da rede modelada como um problema de otimização, conforme mostrado em (Granville et al., 1996) e (Barboza and Salgado, 2001). Neste caso, o mínimo corte de carga numa direção pré-especificada é determinado, de tal forma que uma solução ajustada para as equações da rede incluindo os limites operativos é obtida.

Outras abordagens incluem índices de desempenho alternativos no problema de máximo carregamento. Em (Rosehart, 2000), o FPO é aplicado no cálculo de custos associados ao ponto de demanda máxima. A função objetivo utilizada leva em conta a distância da solução corrente ao ponto de máximo carregamento. Na referência (Kubokawa et al., 2000), a função objetivo é composta pela soma do custo de geração de potência ativa e do parâmetro do carregamento, obtendose simultaneamente uma solução de máxima demanda com mínimo custos de geração de potência ativa.

Neste trabalho, apresenta-se uma formulação do FPO baseada na parametrização da carga e/ou dos limites operativos para evitar a divergência do processo iterativo. A função objetivo é representada pela soma ponderada do parâmetro da carga e/ou dos limites com a função objetivo do problema original. A análise do parâmetro da demanda e/ou limites fornece uma indicação das potenciais causas da divergência do processo iterativo e das possíveis medidas que podem ser adotadas para evitar a divergência. Se o suprimento do carregamento especificado for viável, o algoritmo proposto determina a solução do problema original. Resultados numéricos obtidos com sistemas de pequeno e médio porte ilustram a aplicação da metodologia apresentada.

\section{FORMULAÇÃO BÁSICA}

\subsection{A Superfície Limite de Factibilidade}

Considere o problema de otimização que modela o ajuste da demanda para a restauração da solubilidade das equações da rede elétrica em regime permanente sob condições operativas (Barboza and Salgado, 2001); isto é,

$$
\begin{array}{cc}
\text { Minimizar } & \rho \\
\text { sujeito a } & \mathbf{g}(\mathbf{x}, \rho)=\mathbf{0} \\
& \mathbf{h}(\mathbf{x}) \geq \mathbf{0}
\end{array}
$$

onde, $\rho$ é um escalar denominado parâmetro de carga; $\mathbf{x}$ é o vetor das variáveis de otimização; e $\mathbf{g}(\mathbf{x}, \rho)$ é o vetor das restrições de igualdade parametrizadas por $\rho ; \mathbf{h}(\mathbf{x})$ é o vetor das restrições de desigualdade. As restrições de igualdade representam as equações de balanço de potência nas barras e as restrições de desigualdade expressam os limites das variáveis de otimização e funcionais.

No problema expresso pela equação (1), as componentes do vetor $\mathbf{g}(\mathbf{x}, \rho)$ representam as equações parametrizadas do balanço de potência de cada barra e são expressas por

$$
\begin{gathered}
\triangle P_{j}(\mathbf{x}, \rho)=P_{j}(\mathbf{x})+\left(P_{d 0 j}-\rho \triangle P_{d j}\right) \\
\triangle Q_{j}(\mathbf{x}, \rho)=Q_{j}(\mathbf{x})+\left(Q_{d 0 j}-\rho \triangle Q_{d j}\right)
\end{gathered}
$$

onde, $P_{d 0 j}$ e $Q_{d 0 j}$ são as demandas base de potência ativa e reativa, respectivamente, da barra $j$; e $\triangle P_{d j}$ e $\triangle Q_{d j}$ são as direções do ajuste de carga na barra $j$. Esses valores são supostos conhecidos e estão relacionados qualitativa e quantitativamente a disponibilidade de cada barra para o corte de carga.

As condições de otimalidade de primeira ordem do problema (1) são:

$$
\begin{gathered}
\nabla_{x} \mathbf{g}(\mathbf{x}, \rho)^{t} \lambda+\nabla_{x} \mathbf{h}(\mathbf{x})^{t} \pi=\mathbf{0} \\
\mathbf{g}(\mathbf{x}, \rho)=\mathbf{0} \\
\mathbf{h}_{\in \mathbf{A}(\mathbf{x})=\mathbf{0}} \\
\lambda^{t} \frac{\partial \mathbf{g}(\mathbf{x}, \rho)}{\partial \rho}+1=0
\end{gathered}
$$

onde, $\lambda$ é o vetor dos multiplicadores de Lagrange; $\nabla_{x} \mathbf{g}(\mathbf{x}, \rho)$ é a matriz Jacobiana das equações que representam as restrições de igualdade; $\pi_{i}$ é o multiplicador dual da restrição $i ; \nabla_{x} \mathbf{h}(\mathbf{x})$ é a matriz Jacobiana das equações que representam as restrições de desigualdade e $\mathbf{h}_{\in \mathbf{A}}$ representa o conjunto de restrições de desigualdade ativas no ponto ótimo. 
A primeira das equações (3) pode ser re-escrita como:

$$
\left[\begin{array}{c}
\nabla_{x} \mathbf{g}(\mathbf{x}, \rho) \\
\nabla_{x} \mathbf{h}_{\in \mathbf{A}}(\mathbf{x})
\end{array}\right]^{t}\left[\begin{array}{c}
\lambda \\
\pi
\end{array}\right]=\mathbf{J}_{e}(\mathbf{x}, \rho)^{t} \boldsymbol{\Lambda}=\mathbf{0}
$$

onde, $\mathbf{J}_{e}(\mathbf{x}, \rho)$ é a matriz Jacobiana das equações que representam o conjunto de restrições de igualdade e desigualdade ativas na solução ótima e $\boldsymbol{\Lambda}$ representa o vetor de multiplicadores de Lagrange e duais.

Se nenhum limite é atingido na solução ótima, as condições de otimalidade representadas pela equação (3) são similares às condições de transversalidade da bifurcação de sela-nó das equações do fluxo de potência convencional, exceto pela ordem das matrizes envolvidas. A condição expressa pela primeira equação do conjunto (3) se transforma em

$$
\nabla_{x} \mathbf{g}(\mathbf{x}, \rho)^{t} \lambda=\mathbf{0}
$$

onde a não nulidade dos multiplicadores de Lagrange implica na dependência linear das linhas da matriz $\nabla_{x} \mathbf{g}(\mathbf{x}, \rho)$. Esses multiplicadores podem ser interpretados como colineares com o autovetor à esquerda associado ao autovalor nulo da matriz $\nabla_{x} \mathbf{g}(\mathbf{x}, \rho)$.

Quando existem restrições ativas na solução ótima, o conjunto de equações (4) caracteriza um ponto de bifurcação do conjunto de equações (3), induzida por limites (Greene, 1998; Rosehart, 2000). Esta condição é semelhante aquela da bifurcação sela-nó das equações do fluxo de potência, modificadas quando se atinge um limite operativo (de reativo gerado, por exemplo). A equação (4) estabelece as linhas das matrizes Jacobianas $\nabla_{x} \mathbf{g}(\mathbf{x}, \rho)$ e $\nabla_{x} \mathbf{h}_{\in} \mathbf{A}(\mathbf{x}, \rho)$ são linearmente dependentes. A solução ótima se situa na fronteira da região das soluções factíveis, com o vetor dos multiplicadores de Lagrange sendo acrescido dos componentes duais correspondentes às restrições ativas.

Aspectos da não nulidade do vetor à esquerda e da dependência linear das linhas da matriz Jacobiana das restrições de igualdade e desigualdade ativas são descritos por Greene em (Greene, 1998; Greene et al., 2002). Desde que a esta matriz não é quadrada, utiliza-se o conceito de vetor à esquerda calculado na solução ótima em substituição ao autovetor à esquerda associado ao autovalor nulo da matriz Jacobiana das equações que representam o problema de fluxo de potência convencional. A não nulidade do vetor à esquerda indica a dependência linear das equações da rede com os limites operativos e permite identificar um vetor normal à hipersuperfície limite da região das soluções viáveis do fluxo de potência, de forma similar aquela mostrada em (Dobson, 1992).

$\mathrm{O}$ vetor $\boldsymbol{\Lambda}$ define uma hipersuperfície limite do conjunto factível caracterizado pela dependência linear do gradiente do conjunto das restrições de igualdade e desigualdade. No caso do fluxo de potência via Newton-Raphson, $\boldsymbol{\Lambda}$ é o autovetor à esquerda correspondente ao autovalor nulo da matriz Jacobiana das equações da rede, sendo utilizado para se ajustar a carga para a obtenção de uma solução real. Além disso, este vetor define a direção de mínima distância à hipersuperfície limite de solubilidade das equações do fluxo de potência. De forma similar, no problema de otimização restrito esse vetor indica as cargas com maior influência no máximo carregamento sob condições operativas.

Os multiplicadores duais são associados a sensibilidade do parâmetro $\rho$ em relação aos limites atingidos, fornecendo uma idéia de como estas grandezas podem ser ajustados para melhorar o valor da função objetivo. Da equação (4), os multiplicadores duais estão relacionados aos multiplicadores de Lagrange segundo a equação.

$$
\lambda=-\left(\nabla_{x} \mathbf{g} \nabla_{x} \mathbf{g}^{t}\right)^{-1} \nabla_{x} \mathbf{g}^{t}\left(\nabla_{x} \mathbf{h}^{t} \pi\right)=\mathbf{G} \pi
$$

onde $\mathbf{G}$ é a matriz de sensibilidade que relaciona os valores dos multiplicadores de Lagrange e duais.

A equação (5) expressa uma dependência entre o balanço de potência e os limites operativos. Isto mostra que as medidas corretivas a serem tomadas para se obter uma solução operacional podem ser determinadas modificando-se a demanda de potência e/ou os limites.

\subsection{O Caso Geral do FPO}

Considere o problema de otimização restrita da forma

$$
\begin{array}{cc}
\text { Minimizar } & f_{o}(\mathbf{x}) \\
\text { sujeito a } & \mathbf{g}(\mathbf{x})=\mathbf{0} \\
& \mathbf{h}(\mathbf{x}) \geq \mathbf{0}
\end{array}
$$

onde $f_{o}(\mathbf{x})$ é o índice de desempenho a ser otimizado e os outros termos foram previamente definidos.

As condições de otimalidade do problema (6) são dadas por

$$
\begin{aligned}
\nabla_{x} f_{o}(\mathbf{x})+\lambda^{t} \nabla_{x} \mathbf{g}(\mathbf{x})+\pi^{t} \nabla_{x} \mathbf{h}(\mathbf{x}) & =\mathbf{0} \\
\mathbf{g}(\mathbf{x}) & =\mathbf{0} \\
\mathbf{h}_{\in \mathbf{A}}(\mathbf{x}) & =\mathbf{0} \\
\pi_{i} & \leq 0
\end{aligned}
$$

onde $\nabla_{x} f_{o}(\mathbf{x})$ é o vetor gradiente da função objetivo e os outros termos foram definidos anteriormente.

Hong em (Hong, 1991), propõe uma estratégia para determinar o limiar das variáveis duais no caso de divergência do processo iterativo do FPO. Se a solução é factível, o valor do multiplicador dual requerido para manter a restrição no limite é finito. Quando a solução é não-factível, uma ou mais restrições de igualdade/desigualdade permanecerão violadas, resultando no valor de alguns multiplicadores tendendo a infinito. A estratégia proposta em (Hong, 1991) para tratar o 
problema de inviabilidade da solução FPO consiste em fixar estes multiplicadores num valor limite e relaxar convenientemente as restrições violadas de forma a se alcançar um ponto de equilíbrio. Uma das conclusões importantes de (Hong, 1991) é que, ao tender a infinito o valor de um ou mais multiplicadores a função objetivo assume um valor desprezível em relação ao produto entre o vetor dos multiplicadores de Lagrange e duais e a matriz Jacobiana das restrições de igualdade e desigualdade ativas. No caso do problema expresso pela equação (6), isto pode ser demonstrado dividindo-se a primeira das equações (7) pela norma do vetor $\Lambda$

$$
\frac{\nabla_{x} f_{o}(\mathbf{x})}{\|\Lambda\|}+\left[\begin{array}{c}
\nabla_{x} \mathbf{g}(\mathbf{x}) \\
\nabla_{x} \mathbf{h}_{\in \mathbf{A}}(\mathbf{x})
\end{array}\right]^{t} \frac{\Lambda}{\|\Lambda\|}=\mathbf{0}
$$

Considerando que:

- a norma do vetor $\nabla_{x} f_{o}(\mathbf{x})$ é mantida em geral numa faixa limitada de valores;

- o vetor $\frac{\Lambda}{\|\Lambda\|}$ tem norma unitária;

- ponto crítico alguns multiplicadores de Lagrange e/ou duais apresentam valores muito elevados (Hong, 1991; Almeida and Salgado, 2000),

o primeiro termo da equação (8) pode ser desprezado, o que resulta em

$$
\left[\begin{array}{c}
\nabla_{x} \mathbf{g}(\mathbf{x}, \rho) \\
\nabla_{x} \mathbf{h}_{\in \mathbf{A}}(\mathbf{x})
\end{array}\right]^{t} \frac{\Lambda}{\|\Lambda\|}=\mathbf{0}
$$

Esta equação mostra que o vetor normal à superfície limite da região das soluções factíveis é basicamente definido pelos multiplicadores de Lagrange e duais normalizado. Deve ser observado que os algoritmos de solução do problema (6) apresentam problemas de condicionamento numérico para carregamentos próximos a fronteira da região de factibilidade. Isto não acontece no problema parametrizado (1) porque a quarta condição da equação (3) impõe um limite a esses multiplicadores. Isto implica em que as soluções de máximo carregamento relativas a formulação (1) podem não ser obtidas quando o problema é modelado convencionalmente via equação (6).

\section{USO DE PARAMETRIZAÇÃO}

No presente trabalho, a solução do problema de FPO expresso na equação (6) é determinada considerando-se a parametrização da carga e/ou dos limites operativos. Isto permite evitar a divergência do processo iterativo quando a demanda especificada supera o máximo carregamento operativo. Se a solução do problema de otimização está situada no interior da região definida por estes limites, a solução convencional do problema (6) é determinada.

Este tipo de formulação pode ser aplicado numa variedade de casos práticos. Por exemplo, quando ocorrem contingências no sistema de transmissão, em geral a capacidade de carregamento do sistema é reduzida, tal que a convergência do processo iterativo do problema (6) é dificultada por causa do excesso de demanda. A solução do problema (1) resulta num despacho de potência obtido em função da minimização do parâmetro da carga. Isto requer o reajuste dos controles via solução do problema (6) com a demanda modificada. Neste caso, não é possível garantir a convergência do FPO porque a solução obtida para o problema (1) está situada na fronteira da região das soluções viáveis, e neste ponto crítico a matriz Jacobiana das restrições de igualdade e desigualdade ativas tem posto deficiente (Almeida and Galiana, 1995).

Quando apenas os limites são parametrizados, o carregamento do sistema é mantido porém os limites préespecificados são relaxados para que a convergência seja alcançada. Isto permite solucionar problemas nos quais o carregamento é inferior aquele correspondente ao ponto de bifurcação sela-nó das equações do fluxo de potência e fornece subsídios para se modelar a região de operação da rede elétrica nas situações de emergência.

\subsection{A Parametrização da Demanda}

O problema de FPO com carga parametrizada pode ser expresso como

$$
\begin{array}{cc}
\text { Minimizar } & \omega \rho+f_{o}(\mathbf{x}) \\
\text { sujeito a } & \mathbf{g}(\mathbf{x}, \rho)=\mathbf{0} \\
& \mathbf{h}(\mathbf{x}) \geq \mathbf{0} \\
& \rho \geq \mathbf{0}
\end{array}
$$

onde, as equações e variáveis têm o mesmo significado que nos problemas (6) e (1) e $\omega$ é um fator de ponderação. O parâmetro da carga é limitado, de forma que quando a solução se encontra dentro da região definida pelas equações de balanço de potência e pelos limites operativos, o valor do parâmetro $\rho$ é nulo. Neste caso, a solução obtida coincide com a do problema (6).

A formulação do problema (9) é semelhante àquela apresentada na referência (Kubokawa et al., 2000). As principais diferenças entre essas duas abordagens são a inclusão do fator $\omega$ e o tipo de solução obtida. Kubokawa em (Kubokawa et al., 2000) busca um despacho que maximiza a distância da solução viável corrente ao ponto de máximo carregamento e minimiza simultaneamente o custo de geração de potência ativa. No presente trabalho, não se considera a disponibilidade de uma solução viável a priori. Além disso, as características do problema original são modificadas apenas se a 
convergência para a solução do FPO com carregamento original especificado não for possível.

As condições de otimalidade de primeira ordem do problema representado pela equação (9) são dadas por

$$
\begin{aligned}
\nabla_{x} f_{o}(\mathbf{x})+\nabla_{x} \mathbf{g}(\mathbf{x}, \rho)^{t} \lambda+\nabla_{x} \mathbf{h}(\mathbf{x})^{t} \pi & =\mathbf{0} \\
\mathbf{g}(\mathbf{x}, \rho) & =\mathbf{0} \\
\mathbf{h}(\mathbf{x}) & \geq \mathbf{0} \\
\frac{\partial \mathbf{g}(\mathbf{x}, \rho)}{\partial \rho} \lambda+\pi_{\rho}+\omega & =0 \\
\rho & \geq 0 \\
\pi_{i} & \leq 0
\end{aligned}
$$

onde $\pi_{\rho}$ representa o multiplicador associado à restrição de não negatividade do parâmetro de carga.

Na equação (10) é possível observar que:

- se $\rho=0, \pi_{\rho}<0$, tal que não é necessário o corte de carga. Neste caso, as soluções dos problemas (9) e (6) são iguais, ambas situadas no interior da região definida pelas equações de balanço de potência e pelos limites operativos originais. $\mathrm{O}$ multiplicador correspondente à restrição de não negatividade é expresso como

$$
\pi_{\rho}=-\frac{\partial \mathbf{g}(\mathbf{x}, \rho)}{\partial \rho} \lambda-\omega
$$

- se $\rho>0, \pi_{\rho}=0$, o corte de carga é necessário, e neste caso a solução está localizada na fronteira da região das soluções viáveis. Da equação (11),

$$
\frac{\partial \mathbf{g}(\mathbf{x}, \rho)}{\partial \rho}(\lambda / \omega)=-1
$$

Seja a primeira das equações (10) ponderada pelo fator $\omega$; isto é,

$$
\frac{1}{\omega} \nabla_{x} f_{o}(\mathbf{x})+\nabla_{x} \mathbf{g}(\mathbf{x}, \rho)^{t} \frac{\lambda}{\omega}+\nabla_{x} \mathbf{h}(\mathbf{x})^{t} \frac{\pi}{\omega}=\mathbf{0}
$$

Para que a solução obtida pertença à fronteira da região das soluções viáveis é necessário que

$$
\frac{1}{\omega} \nabla_{x} f_{o}(\mathbf{x}) \rightarrow \mathbf{0}
$$

o que requer $\left\|\nabla_{x} f_{o}(\mathbf{x})\right\| \ll \omega$. Se esta condição for satisfeita, então a solução obtida através do ajuste da demanda é a mesma do problema (1).

\subsection{A Parametrização dos Limites}

A parametrização dos limites operativos é representada analiticamente pelo seguinte problema de otimização:

$$
\begin{array}{cc}
\text { Minimizar } & \omega \rho+f_{o}(\mathbf{x}) \\
\text { sujeito a } & \mathbf{g}(\mathbf{x})=\mathbf{0} \\
& \mathbf{h}(\mathbf{x}, \rho) \geq \mathbf{0} \\
& \rho \geq \mathbf{0}
\end{array}
$$

onde

$$
\mathbf{h}(\mathbf{x}, \rho)=\mathbf{h}(\mathbf{x})-\rho \Delta \mathbf{h}^{e}
$$

é um vetor de funções que representam os limites operativos. As equações $\mathbf{h}(\mathbf{x})$ são as mesmas do problema (6), acrescidas dos valores pré-determinados dos incrementos nos limites $\Delta \mathbf{h}^{e}$ parametrizados pelo escalar $\rho$.

A restrição de não-negatividade evita valores de $\rho$ que impliquem numa redução da faixa imposta pelos limites. Se a solução do problema expresso pela equação (6) pertence à região definida pelas restrições de igualdade e de desigualdade originais, então $\rho=0$. Um valor positivo de $\rho$ implica em que os limites devem ser relaxados para que se obtenha a convergência do processo iterativo. Neste caso, a solução determinada é um ponto pertencente à fronteira definida pelos limites operativos relaxados.

As condições de otimalidade de primeira ordem para problema expresso pela equação (15) são:

$$
\begin{aligned}
\nabla_{x} f_{o}(\mathbf{x})+\nabla_{x} \mathbf{g}(\mathbf{x})^{t} \lambda+\nabla_{x} \mathbf{h}(\mathbf{x}, \rho)^{t} \pi & =\mathbf{0} \\
\mathbf{g}(\mathbf{x}) & =\mathbf{0} \\
\mathbf{h}(\mathbf{x}, \rho) & \geq \mathbf{0} \\
\frac{\partial \mathbf{h}^{t}(\mathbf{x}, \rho)}{\partial \rho} \pi+\pi_{\rho}+\omega & =0 \\
\rho & \geq 0 \\
\pi_{i} & \leq 0
\end{aligned}
$$

onde os todos os termos foram previamente definidos.

A análise das condições de otimalidade do problema (15) indica que as três primeiras equações são semelhantes às condições estabelecidas para o problema (6), diferindo apenas quanto ao valor dos multiplicadores duais. O módulo do vetor destes multiplicadores deve satisfazer a quarta condição representada pela equação (17); isto é,

$$
\frac{\partial \mathbf{h}^{t}(\mathbf{x}, \rho)}{\partial \rho}=-\Delta \mathbf{h}^{e}
$$

Conforme pode ser inferido desta equação, o valor dos multiplicadores depende da direção pré-especificada de relaxamento dos limites. Quando existe uma solução que satisfaz os limites operativos, $\rho=0 \mathrm{e}$

$$
\pi_{\rho}=\Delta \mathbf{h}^{e^{t}} \pi-\omega<0
$$


e os multiplicadores duais $\pi$ são correspondentes aos do problema (6).

Caso não exista solução que satisfaça os limites operativos, $\rho>0, \pi_{\rho}=0$ e o valor dos multiplicadores duais satisfaz a relação

$$
\Delta \mathbf{h}^{e^{t}} \pi=\omega
$$

Similarmente ao caso da demanda parametrizada, é possível mostrar que a solução obtida com os limites relaxados pertence à fronteira da nova região de factibilidade definida à partir dos limites relaxados. A condição que deve satisfazer $\omega$, da mesma forma que no caso da redução da demanda é

$$
\left\|\nabla_{x} f_{o}\right\| \ll \omega
$$

A parametrização dos limites requer a inclusão de uma restrição de não negatividade no problema expresso pela equação (15) para cada tipo de limite sujeito a relaxação. Cada tipo de restrição está associado a um parâmetro $\rho$ e cada restrição sujeita a relaxação está associada a um fator $\Delta h_{i}^{e}$.

Deve ser observado, que a ausência de um limite superior para o parâmetro $\rho$ implica em que as restrições selecionadas serão relaxadas o quanto for preciso. Se esta relaxação não for suficiente para se obter uma solução das equações da rede elétrica, isto indica que o ponto crítico (de bifurcação sela-nó) das equações do fluxo de potência convencional foi ultrapassado. Neste caso, o corte de carga se torna necessário como uma alternativa (ou uma medida corretiva adicional) a relaxação dos limites.

\section{RESULTADOS NUMÉRICOS}

Para ilustrar a aplicação da metodologia proposta, resultados numéricos obtidos com sistemas teste de 6 e 118 barras e com um equivalente do sistema sul-sudeste brasileiro de 750 barras são apresentados. O problema de otimização é resolvido através do método não-linear primal-dual de pontos interiores, versão preditor-corretor, implementado no ambiente Matlab 6.0. Os testes realizados visaram analisar a robustez da metodologia proposta em termos de convergência do processo iterativo. O procedimento adotado é resumido nos seguintes passos:

- à partir de uma carga base, determinou-se o ponto de máximo carregamento operacional de cada um dos sistemas teste através do programa computacional baseado em (Irisarri et al., 1997);

- um carregamento superior aquele obtido no item anterior foi especificado, buscando-se uma solução do FPO com o aplicativo computacional descrito em (Flupot,
1997). Isto resultou na não convergência do processo iterativo do FPO convencional;

- a metodologia proposta foi utilizada para se obter uma solução operacional, através do ajuste da demanda e/ou dos limites operacionais. Os limites foram modelados segundo a equação (16), considerando-se dois parâmetros, um correspondente aos limites de magnitude da tensão (denotado $\rho_{V}$ ) e outro relativo aos limites de potência ativa e reativa (denotado $\rho_{P Q}$ ).

- com base no carregamento para o qual não há solução do FPO e na solução obtida no item anterior, utilizouse o programa computacional baseado em (Barboza and Salgado, 2001) para determinar o máximo carregamento operacional;

- a solução determinada via metodologia proposta foi validada com o programa computacional descrito em (Flupot, 1997).

\subsection{Sistema de 6 barras}

Os dados deste sistema foram obtidos em (Pai, 1979). Os limites de magnitude de tensão e taps são: 0,90 pu a $1,10 \mathrm{pu}$; geração de potência ativa: 0,0 a $210 \mathrm{MW}$ e 0,0 a $60 \mathrm{MW}$ e geração de potência reativa: $-20,0$ a 95,0 Mvar e -15,0 a 70 Mvar. O ponto de máximo carregamento operacional deste sistema corresponde a um acréscimo de $32 \%$ na carga base.

O aumento de $50 \%$ na demanda base, com o fator de potência constante, corresponde a um carregamento de $82,5 \mathrm{MW}$ e 19,5 Mvar na barra 3; 45,0 MW e 27,0 Mvar na barra 5; e 75,0 MW e 7,5 Mvar na barra 6, totalizando 202,5 MW e 54,0 Mvar no sistema. Não existe solução operacional para este nível de carregamento e por isto o processo iterativo do FPO convencional diverge.

\subsubsection{Parametrização da carga}

Neste caso, o índice otimizado é o custo de geração de potência ativa, com coeficientes unitários para a curva de custo de geração e fator de ponderação $\omega$ igual a 10000.

A Tabela (1) apresenta os resultados obtidos com a metodologia proposta. As colunas mostram respectivamente, o índice das barras, o módulo da tensão complexa, os despachos de potência ativa e reativa dos geradores, a demanda ajustada e os multiplicadores de Lagrange.

O aplicativo computacional baseado em (Barboza and Salgado, 2001) foi aplicado na demanda para a qual não há solução operacional. O mesmo ajuste na demanda foi obtido nas duas simulações, com o parâmetro de carga assumindo o valor 0,1378 . O despacho de potência dos geradores é seme- 
Tabela 1: Sistema de 6 barras - ajuste da demanda / mínimo custo de geração

\begin{tabular}{||c|c|c|c|c|c|c|c||}
\hline \hline $\mathrm{N}$ & $\begin{array}{c}V \\
\mathrm{pu}\end{array}$ & $\begin{array}{c}P_{g} \\
\mathrm{MW}\end{array}$ & $\begin{array}{c}Q_{g} \\
\text { Mvar }\end{array}$ & $\begin{array}{c}P_{d} \\
\mathrm{MW}\end{array}$ & $\begin{array}{c}Q_{d} \\
\text { Mvar }\end{array}$ & $\begin{array}{c}\lambda_{p} \\
\$ / \mathrm{MW}\end{array}$ & $\begin{array}{c}\lambda_{q} \\
\$ / \text { Mvar }\end{array}$ \\
\hline \hline 1 & 1,10 & 149,3 & 73,2 & 0,0 & 0,0 & - & - \\
\hline 2 & 1,10 & 44,8 & 33,2 & 0,0 & 0,0 & - & - \\
\hline 3 & 0,90 & 0,0 & 0,0 & 72,1 & 17,0 & 2693,6 & 3661,0 \\
\hline 4 & 0,93 & 0,0 & 0,0 & 0,0 & 0,0 & 2007,1 & 3487,0 \\
\hline 5 & 0,90 & 0,0 & 0,0 & 39,3 & 23,6 & 4306,6 & 6917,3 \\
\hline 6 & 0,90 & 0,0 & 0,0 & 65,5 & 6,5 & 3698,1 & 6460,8 \\
\hline \hline & & 194,1 & 106,4 & 176,9 & 47,1 & & \\
\hline \hline
\end{tabular}

lhante e os vetores dos multiplicadores de Lagrange correspondentes às soluções obtidas através do algoritmo proposto (ver colunas 7 e 8 da Tabela (1)) e do algoritmo de mínimo corte de carga (balanço de potência ativa: 0,$27 ; 0,20 ; 0,43$ e 0,37 ; balanço de potência reativa: 0,$37 ; 0,35 ; 0,69$ e 0,65 ) apontam na mesma direção. Os multiplicadores correspondentes à potência reativa demandada têm valores superiores aqueles correspondentes à potência ativa demandada, o que indica que a sensibilidade do carregamento crítico é maior com relação à distribuição de potência reativa. $\mathrm{O}$ limite de potência reativa gerada não é atingido, sendo portanto a instalação de compensadores de reativo a forma de aumentar o carregamento dentro destes limites operativos.

\subsubsection{Parametrização dos limites operativos}

Neste caso, dois índices de desempenho foram considerados na solução do problema expresso pela equação (15): o custo de geração de potência ativa e a soma dos desvios quadráticos da magnitude da tensão do valor de 1,0 pu. O valor do fator de ponderação $\omega$ foi especificado em 10000 e os fatores de relaxação foram selecionados como: $\Delta V_{i}^{e}=0,1 \mathrm{pu}$ e $\Delta P_{i}^{e}=1 \mathrm{MW}$ e $\Delta Q_{i}^{e}=1$ Mvar.

A Tabela (2) mostra os resultados da minimização do custo de geração. Os valores resultantes de $\rho_{V}$ e $\rho_{P Q}$ são 0,9799 e $2,48 \times 10^{-7}$, respectivamente. Estes valores indicam que o limite de tensão das barras de carga foi relaxado até 0,8020 pu $(0,9-0,9799 \times 0,1)($ valor da tensão da barra 5$)$ e que os limites de potência ativa e reativa não sofreram modificações.

Na Tabela (3) são mostrados os resultados da minimização da soma dos desvios quadráticos de tensão, com um fator de ponderação igual a 10,0 . Os valores finais de $\rho_{V}$ e $\rho_{P Q}$ são respectivamente 0,7808 e $0,5559 \mathrm{pu}$, sendo portanto modificados os limites de tensão (barra 5: 0,9 para 0,8219 pu) e potência ativa e reativa geradas (barra 1: $210 \mathrm{MW}$ para 215,56 MW e 95,0 Mvar para 100,56 Mvar; barra 2: $60 \mathrm{MW}$ para 65,56 MW e 70,0 Mvar para 75,56 Mvar).
Tabela 2: Sistema de 6 barras - ajuste dos limites / mínimo custo de geração

\begin{tabular}{||c|c|c|c|c|c|c|c||}
\hline \hline $\mathrm{N}$ & $\begin{array}{c}V \\
\mathrm{pu}\end{array}$ & $\begin{array}{c}P_{g} \\
\mathrm{MW}\end{array}$ & $\begin{array}{c}Q_{g} \\
\text { Mvar }\end{array}$ & $\begin{array}{c}P_{d} \\
\mathrm{MW}\end{array}$ & $\begin{array}{c}Q_{d} \\
\mathrm{Mvar}\end{array}$ & $\begin{array}{c}\lambda_{p} \\
\$ / \mathrm{MW}\end{array}$ & $\begin{array}{c}\lambda_{q} \\
\$ / \mathrm{Mvar}\end{array}$ \\
\hline \hline 1 & 1,10 & 174,9 & 95,0 & 0,0 & 0,0 & 3,2 & 0,0 \\
\hline 2 & 1,10 & 54,2 & 52,2 & 0,0 & 0,0 & 4387,0 & 0,0 \\
\hline 3 & 0,83 & 0,0 & 0,0 & 82,5 & 19,5 & 57096,6 & 69608,7 \\
\hline 4 & 0,89 & 0,0 & 0,0 & 0,0 & 0,0 & 41085,8 & 65323,7 \\
\hline 5 & 0,80 & 0,0 & 0,0 & 45,0 & 27,0 & 59173,1 & 73892,1 \\
\hline 6 & 0,86 & 0,0 & 0,0 & 75,0 & 7,5 & 53881,2 & 69225,4 \\
\hline \hline & & 229,1 & 147,2 & 202,5 & 54,0 & & \\
\hline \hline
\end{tabular}

Tabela 3: Sistema de 6 barras - ajuste dos limites / mínimo desvio quadrático de tensão

\begin{tabular}{||c|c|c|c|c|c|c|c||}
\hline \hline$N^{o}$ & $\begin{array}{c}V \\
\mathrm{pu}\end{array}$ & $\begin{array}{c}P_{g} \\
\mathrm{MW}\end{array}$ & $\begin{array}{c}Q_{g} \\
\text { Mvar }\end{array}$ & $\begin{array}{c}P_{d} \\
\mathrm{MW}\end{array}$ & $\begin{array}{c}Q_{d} \\
\text { Mvar }\end{array}$ & $\begin{array}{c}\lambda_{p} \\
\$ / \mathrm{MW}\end{array}$ & $\begin{array}{c}\lambda_{q} \\
\$ / \mathrm{Mvar}\end{array}$ \\
\hline \hline 1 & 1,10 & 168,8 & 100,6 & 0,0 & 0,0 & 0,00 & 0,00 \\
\hline 2 & 1,10 & 59,7 & 44,4 & 0,0 & 0,0 & 0,00 & 0,00 \\
\hline 3 & 0,86 & 0,0 & 0,0 & 82,5 & 19,5 & 57,69 & 70,11 \\
\hline 4 & 0,88 & 0,0 & 0,0 & 0,0 & 0,0 & 41,24 & 66,52 \\
\hline 5 & 0,82 & 0,0 & 0,0 & 45,0 & 27,0 & 59,07 & 71,84 \\
\hline 6 & 0,85 & 0,0 & 0,0 & 75,0 & 7,5 & 54,19 & 71,26 \\
\hline \hline & & 228,5 & 145,0 & 202,5 & 54,0 & & \\
\hline \hline
\end{tabular}

\subsection{Sistema de 118 Barras}

O ponto crítico de carregamento do sistema de 118 barras corresponde a um acréscimo de $51 \%$ na demanda base mantendo-se o fator de potência constante. A adoção de um aumento de $80 \%$ na demanda resulta na divergência do processo iterativo do FPO. Os coeficientes das curvas de custo dos geradores, apresentados na Tabela (4), estão situados na mesma faixa de valores, exceto aqueles correspondentes a barra 12, para os quais foram adotados valores consideravelmente superiores aos das outras barras.

Tabela 4: Sistema de 118 barras - Coeficientes de custo dos geradores

\begin{tabular}{||c|c|c||c|c|c||c|c|c||}
\hline \hline No. & $C L$ & $C Q$ & No. & $C L$ & $C Q$ & No. & $C L$ & $C Q$ \\
\hline 4 & 2,00 & 1,00 & 49 & 0,97 & 2,50 & 89 & 0,53 & 0,53 \\
\hline 8 & 1,00 & 1,00 & 54 & 0,90 & 3,50 & 90 & 0,23 & 5,00 \\
\hline 10 & 0,97 & 0,75 & 59 & 0,53 & 5,25 & 91 & 0,23 & 4,00 \\
\hline 12 & 60,00 & 7,50 & 61 & 0,53 & 5,25 & 99 & 0,23 & 3,00 \\
\hline 24 & 3,00 & 2,50 & 65 & 0,30 & 1,87 & 100 & 0,60 & 2,00 \\
\hline 25 & 0,52 & 2,00 & 66 & 0,90 & 0,75 & 103 & 0,90 & 3,50 \\
\hline 26 & 0,30 & 1,87 & 69 & 0,90 & 0,75 & 107 & 1,57 & 2,50 \\
\hline 27 & 2,00 & 1,75 & 72 & 2,00 & 7,50 & 111 & 0,90 & 3,50 \\
\hline 31 & 0,80 & 4,50 & 73 & 2,75 & 6,50 & 112 & 4,50 & 2,50 \\
\hline 40 & 2,50 & 4,50 & 80 & 0,90 & 1,50 & 113 & 1,50 & 2,50 \\
\hline 42 & 2,50 & 4,50 & 87 & 2,50 & 1,50 & 116 & 1,85 & 3,50 \\
\hline 46 & 0,90 & 3,50 & & & & & & \\
\hline \hline
\end{tabular}




\subsubsection{Seleção do Fator de Ponderação}

Visando analisar a qualidade das soluções obtidas através da metodologia proposta com relação ao valor do fator de ponderação $\omega$, cinco valores foram atribuídos a este fator $(1,10$, 100,1000 e 10000) na minimização do custo de geração. O carregamento foi especificado no nível mencionado anteriormente.

A Tabela (5) mostra o efeito da variação do fator de ponderação quando o custo de geração de potência ativa é minimizado, admitindo-se a possibilidade de um ajuste na carga. Nota-se que a redução deste fator aumenta a importância do ajuste de carga no processo de otimização. A variação de $\omega$ desde 1 até 10000 resulta em que o gradiente da função objetivo $\left(\nabla f_{o}\right)$, o parâmetro de ajuste da demanda ( $\rho$ e o valor de mínimo custo de geração tendem a um valor constante). Os coeficientes da curva de custo de geração se tornam insignificantes em comparação com o valor de $\omega$, o que resulta na priorização de ajustar a demanda ao invés de despachar economicamente os geradores.

Tabela 5: Sistema de 118 barras com carga parametrizada Variação do fator de ponderação $\omega$ - ajuste da demanda

\begin{tabular}{||c|c|c|c|c|c||}
\hline \hline$\omega$ & $\nabla f_{o}$ & $\rho$ & $V^{\min }$ & $N^{o}$ & $\$$ \\
\hline 1 & 69,94 & 0,9490 & 0,900 & 26 & 138,27 \\
\hline 10 & 72,68 & 0,3781 & 0,900 & 26 & 19087,31 \\
\hline 100 & 85,03 & 0,1615 & 0,900 & 76 & 30784,98 \\
\hline 1000 & 85,64 & 0,1612 & 0,900 & 76 & 31484,40 \\
\hline 10000 & 85,77 & 0,1612 & 0,900 & 76 & 31701,24 \\
\hline \hline
\end{tabular}

Quando $\omega=1$ e $\omega=10$, o ajuste da demanda não é preponderante e por esta razão a demanda é reduzida em 94,9\% e $37,8 \%$ respectivamente, o que resulta num custo de geração consideravelmente reduzido. A barra 26 (geração) apresenta a mínima magnitude de tensão. Quando $\omega=100$, a prioridade do ajuste da demanda é aumentada, de forma que o ajuste na demanda é menor do que o caso anterior. Nos casos em que $\omega=1000$ e $\omega=10000$, esta tendência se acentua de forma que a diminuição na demanda tende a ser mínima, com todos os geradores despachados no seu limite máximo.

A Tabela (6) resume os resultados obtidos com a variação do fator de ponderação no caso em que os limites são ajustados para se obter uma solução do FPO. No caso em questão, o aumento deste fator reforça o efeito do ajuste dos limites no processo de otimização. A consequiência da variação de $\omega$ desde 1 até 10000 é que o gradiente da função objetivo $\left(\nabla f_{o}\right)$, os parâmetros de ajuste dos limites $\left(\rho_{V}\right.$ e $\left.\rho_{P Q}\right)$ e a mínima magnitude da tensão $\left(V^{\text {min }}\right)$ tendem a um valor constante. Os coeficientes custo se tornam insignificantes em comparação com o valor de $\omega$, o que resulta na priorização de se minimizar o ajuste nos limites sobre o despacho econômico dos geradores no processo de otimização.
Tabela 6: Sistema de 118 barras com limites parametrizados - Variação do fator de ponderação $\omega$ - ajuste dos limites

\begin{tabular}{||c|c|c|c|c|c|c||}
\hline \hline$\omega$ & $\nabla f_{o}$ & $\rho_{V}$ & $\rho_{P Q}$ & $V^{m i n}$ & $\mathrm{~N}$ & $\$$ \\
\hline 1 & 88,37 & 0,8017 & 10,02 & 0,819 & 76 & 45985,00 \\
\hline 10 & 91,45 & 0,5071 & 4,32 & 0,849 & 76 & 48420,00 \\
\hline 100 & 94,08 & 0,4676 & 2,33 & 0,850 & 76 & 51723,00 \\
\hline 1000 & 106,49 & 0,4579 & 1,894 & 0,854 & 76 & 58648,00 \\
\hline 10000 & 106,49 & 0,4572 & 1,894 & 0,853 & 76 & 58650,00 \\
\hline \hline
\end{tabular}

A análise dos limites de potência ativa relaxados para diferentes valores de $\omega$ mostra a peculiaridade do comportamento da barra 12 (a de custo de geração mais elevado). Esta unidade seria despachada apenas quando os outros geradores, localizados mesma área, atingissem os seus limites máximos de potência ativa gerada.

No caso em que $\omega=1$, o ajuste dos limites não é preponderante na otimização do função multiobjetivo. No segundo caso, a prioridade do ajuste dos limites é aumentada, de forma que uma relaxação nos limites menor do que a do caso anterior é determinada. Isto resulta em que a geração menos econômica (barras 24, 111 e 113) é despachada no seu limite máximo. Nos outros três casos, esta tendência se acentua de forma que a relaxação tende a ser mínima, com todos os geradores despachados no seu limite máximo.

O relaxamento dos limites de reativo gerado não é crítico, nenhum gerador atingindo o seu limite de potência reativa.

Observa-se portanto, que a especificação do fator de ponderação $\omega$ tem considerável influência na qualidade da solução obtida. Para assegurar uma abertura mínima dos limites o valor deste fator deve ser superior ao da norma do gradiente da função objetivo selecionada. Se o problema original possui uma solução operativa viável e se $\omega$ for menor que a norma do gradiente da função objetivo, os limites de potência gerada dos geradores com menor custo tenderão a ser relaxados, modificando-se desta forma o problema original. Este fato pode ser utilizado em estudos de planejamento, onde a escolha de baixos fatores de ponderação permitiria determinar os geradores mais econômicos dentro de um conjunto definido previamente. Da mesma forma, uma solução que apresente uma relaxação mínima dos limites de geração mostraria os diferentes custos associados a cada gerador operando no máximo carregamento.

\subsubsection{Parametrização da Demanda}

Neste caso, três funções objetivo foram consideradas: a) o mínimo custo de geração; b) o mínimo desvio quadrático da magnitude da tensão e c) o custo de corte de carga numa direção pré-especificada. Duas situações foram analisadas. Na primeira, o corte de carga foi efetuado apenas nas barras de 
carga, enquanto que no segundo caso a demanda de todas as barras foi considerada disponível para o ajuste. Os resultados numéricos que concernem o custo do corte de carga foram obtidos com o aplicativo computacional descrito na referência (Flupot, 1997).

No caso da minimização do custo de geração, a norma do vetor gradiente da função objetivo na solução ótima é 81,65, valor consideravelmente menor do que o fator de ponderação (especificado em 10000). De forma semelhante, quando se minimiza o desvio quadrático de tensão, $\omega=1000$ e a norma do vetor gradiente é igual a 0,613 no ponto ótimo.

Quando o corte é efetuado apenas nas barras de carga, a demanda que o sistema pode suprir é a mesma no caso da minimização tanto do custo de geração quanto do desvio quadrático da magnitude da tensão. Neste último caso, o valor final do parâmetro de ajuste da carga é 0,16091 pu, o que representa uma redução de 742,64 MW e 290,05 Mvar na demanda, num total de 7425,00 MW e 2590,92 Mvar. Quando o custo de geração de potência ativa é minimizado, o parâmetro de carga assume o valor 0,1638 , o que representa um corte de carga de 756,03 MW e 295,27 Mvar. No caso em que a função objetivo custo de corte de carga numa direção pré-especificada é considerada, o valor do corte é de 16,38\% na demanda a ser originalmente suprida, o que implica numa redução 756,04 MW e 295,28 Mvar.

Os despachos de potência ativa obtidos na otimização desses índices são semelhantes. Os fatores com influência mais acentuada na solução ótima são os limites de tensão e de potência ativa gerada nas barras 76 e 118. Os despachos de potência reativa diferem ligeiramente, em razão da natureza distinta dos índices otimizados.

Quando todas as barras são disponibilizadas para o ajuste na demanda os limites de tensão são críticos, tal que diferentes despachos de potência são obtidos. Quando se otimiza o custo de corte de carga, a demanda máxima que o sistema pode suprir corresponde a um acréscimo de $50,98 \%$ na demanda base. Isto indica que um corte de carga de 1196,6 MW e 417,53 Mvar na demanda especificada é necessário. Para o despacho fornecido por este índice, o custo de geração de potência ativa é de $32624,0 \$$

O custo de geração de potência ativa otimizado é igual a 31484,0 . A obtenção de uma solução ajustada requer um corte de $16,12 \%$ na demanda especificada, o que representa uma redução de 1196,6 MW e 417,56 Mvar na carga.

Quando o desvio quadrático da tensão é minimizado, o custo de geração de potência ativa é de 32977,0\$, sendo necessário um decréscimo de $15,78 \%$ na demanda. Isto representa um corte de carga de potência ativa e reativa de 1171,80 MW e 408,90 Mvar, respectivemente.
A maior diferença nos despachos de potência ativa correspondentes a estes índices de desempenho é a do gerador da barra 12, devido ao seu elevado coeficiente da curva de custo de geração. Quando o desvio quadrático de tensão é minimizado, esta unidade geradora é despachada com o seu valor máximo de potência. No caso da minimização do custo de geração, este gerador não é despachado, devido ao alto custo associado. Por outro lado, devido às distintas naturezas dos índices de desempenho, diferentes despachos de potência reativa são obtidos. Isto confirma que, se o limite de tensão é o fator de maior influência na determinação da demanda máxima, então diferentes soluções podem ser obtidas para um mesmo ajuste na demanda.

\subsection{Sistema de 750 Barras}

O máximo carregamento do equivalente do sistema sulsudeste brasileiro de 750 barras corresponde a um acréscimo de 205\% na carga base. As restrições operativas são: 0 e 500 MW para os limites de potência ativa gerada, 200 Mvar e 200 Mvar para os limites de potência reativa gerada, e 0,90 e 1,10 pu para os limites de tensão e tapes dos transformadores. O índice de desempenho otimizado foi o custo de geração de potência ativa. Para suprir este carregamento, os limites de potência reativa de diversas barras são atingidos, sendo a barra 370 crítica em termos do limite de tensão. Quando o carregamento foi fixado em $230 \%$ da demanda base $(28389,2$ MW e 15669,7 Mvar) o processo iterativo não converge.

\subsubsection{Parametrização da Demanda}

Para a obtenção da solução operacional via ajuste da demanda, o problema de FPO foi resolvido considerando-se um fator de ponderação igual a 5000 (para possibilitar a comparação com os resultados obtidos com o programa computacional descrito em (Flupot, 1997)). Na solução ótima ajustada, a norma do vetor gradiente da função objetivo é de 4,29, valor bastante inferior ao do fator de ponderação. $\mathrm{O}$ parâmetro de ajuste da demanda é igual a 0,0806 , o que implica num corte de carga total de 2287,2 MW e 1262,4 Mvar.

Um número expressivo de geradores (78 num total de 87 ) atinge o limite de potência reativa, o que indica que a causa da divergência do processo iterativo é a ausência de suporte adequado de potência reativa. Isto resulta na modificação do despacho de potência ativa. O custo de geração de potência ativa, correspondente a solução obtida via algoritmo proposto é de $12185,0 \$$.

Para validar os resultados anteriores, utilizou-se o programa computacional descrito na referência (Flupot, 1997), com as funções Custo do Corte de Carga e Custo de Geração sendo simultaneamente otimizadas. $\mathrm{O}$ ajuste na demanda requerido para a obtenção de uma solução operacional foi de 
$7,96 \%$, representando um corte de carga de $2258,1 \mathrm{MW}$ e 1246,0 Mvar. A demanda ajustada requer uma geração total de 26427,4 MW e 16445,9 Mvar, despachada de forma semelhante aquela correspondente à solução obtida através da abordagem proposta. Se o custo de corte de carga é otimizado isoladamente, a demanda requer um ajuste de $8,10 \%$ sobre o carregamento base (corte de carga de 2298,1 MW e $1268,1 \mathrm{Mvar}$ ). A geração requerida passa a ser 26384,8 MW e 16404,2 Mvar, com o despacho do geradores diferindo daqueles obtidos através da metodologia proposta.

Minimizando-se os custos do corte de carga e de geração através do programa descrito na referência (Flupot, 1997), obtém-se um custo de geração de 12186,6 . No caso da minimização isolada do corte de carga, realizada com este mesmo programa computacional, o valor deste custo é de 12111,0\$ (como conseqüência do corte de carga ser $0,14 \%$ superior aquele obtido no caso da soma das funções mencionadas anteriormente). Observe-se que a quantidade total de demanda suprimida através da minimização do custo de corte de carga varia com o fator asociado ao parâmetro da carga.

\subsubsection{Parametrização dos Limites}

Neste caso, os incrementos nos limites foram fixados em 0,1 pu, 10 MW e 10 Mvar. Para a obtenção da solução ajustada, os parâmetros $\rho_{V}$ e $\rho_{P Q}$ assumem respectivamente os valores $4,87 \times 10^{-10}$ e 1,8684 . Portanto, os limites de tensão permanecem constantes enquanto que os limites de potência ativa e reativa geradas são modificados para 518,7 MW e 218,7 Mvar em todas as barras.

Uma vez mais, um grande número de geradores (77 num total de 87) atinge o limite de potência reativa gerada, indicando o suporte de potência reativa como um fator restritivo para a determinação de uma solução operacional. Conseqüentemente, o despacho de potência ativa também é modificado. A ausência de suporte de potência reativa faz com que geradores com maior custo sejam despachados. A dependência da relaxação dos limites de potência ativa e reativa gerada com um único parâmetro resulta em que os limites de potência ativa são relaxados mais do que o necessário.

Para validar a solução determinada através da metodologia proposta, o problema de FPO foi resolvido com os limites relaxados, utilizando-se o programa computacional descrito em (Flupot, 1997). Os índices de desempenho utilizados foram o carregamento e o custo de geração. Quando o carregamento é maximizado isoladamente, o resultado obtido difere em $0,5 \%$ com relação à solução determinada via metodologia proposta. No caso do custo de geração, a convergência é obtida para a demanda especificada ( $230 \%$ da demanda base).

\section{CONCLUSÕES}

O problema de FPO pode ser formulado parametrizando-se a demanda e/ou limites operativos. Isto evita a divergência do processo iterativo e fornece soluções situadas na fronteira da região das soluções viáveis operativas. A parametrização da carga e/ou limites operativos requer a especificação da direção em que a demanda e/ou os limites são ajustados.

$\mathrm{O}$ uso de um fator de ponderação para balancear as funções objetivo desempenha um papel essencial na qualidade das soluções obtidas. A escolha adequada deste fator permite priorizar o ajuste na demanda/limites visando uma relaxação mais reduzida.

O esforço computacional requerido para a aplicação da metodogia proposta é similar ao do fluxo de potência ótimo convencional. Quando o ajuste não é necessário, a abordagem proposta fornece a mesma solução que o algoritmo convencional e não acrescenta esforço computacional significativo.

A aplicação de parametrização aumenta a robustez do FPO, permitindo a obtenção de soluções diferentes para distintas funções objetivos na fronteira da região de factibilidade.

Os resultados numéricos obtidos com sistemas de médio porte mostram o potencial da metodologia proposta para melhorar o desempenho de aplicativos computacionais comerciais.

\section{AGRADECIMENTOS}

C. F. Moyano tem suporte financeiro do Conselho Nacional de Desenvolvimento Científico e Tecnoógico - CNPq / Brazil.

\section{REFERÊNCIAS}

Almeida, K. C. and Galiana, F. D. (1995). Critical cases in the optimal power flow., IEEE Transactions on Power Systems 11(3): 1509-1518.

Almeida, K. C. and Salgado, R. (2000). Optimal power flow solutions under variable load conditions, IEEE Transactions on Power Systems 15(4): 1204-1211.

Barboza, L. V. and Salgado, R. (2001). Restoring solutions for unsolvable cases via minimum load shedding for a specified direction., Proceedings of the IEEE PICA Conference Proceedings.

Dobson, I. (1992). Observations on the geometry of saddle node bifurcation and voltage collapse in electrical power systems, IEEE Transactions on Circuits and Systems - I Fundamental Theory and Applications 39(3): 559-565. 
Flupot (1997). Programa de Fluxo de Potência Ótimo (FLUPOT) - V5.1/00, Manual do Usuário, Centro de Pesquisas de Energia Elétrica - CEPEL.

Granville, S., Mello, J. C. O. and Melo, A. C. G. (1996). Application of interior point methods to power flow unsolvability., IEEE Transactions on Power Systems 11(2): 1096-1103.

Greene, S. (1998). Margin and sensitivity methods for security analysis of electrical power systems. Doctor of Philosophy Thesis, University of Wisconsin-Madison.

Greene, S., Dobson, I. and Alvarado, F. L. (2002). Sensitivity of transfer capability margins with a fast formula, IEEE Transactions on Power Systems 17(1): 34-40.

Hong, Y.-Y. (1991). Clarification of the applicability of infeasibility treatment for the newton optimal power flow method, Electric Power System Research 21: 1-7.

Irisarri, G. D., Wang, X., Tong, J. and Mokhtari, S. (1997). Maximum loadability of power systems using non linear interior point method, IEEE Transactions on Power Systems 12(1): 162-172.

Kubokawa, J., Inoue, R. and Sasaki, H. (2000). A solution of optimal power flow with voltage stability constrains, IEEE Transactions on Power Systems pp. 625-630.

Pai, M. A. (1979). Computer Techniques in Power System Analysis, Tata McGraw-Hill Publishing Company Limited.

Rosehart, W. (2000). Optimization of power systems with voltage security constrains. PhD Thesis - University of Waterloo -Department of Electrical Engineering - Waterloo - Canada. 